Towards a high-resolution flow camera using artificial hair sensor arrays for flow pattern observations

This article has been downloaded from IOPscience. Please scroll down to see the full text article.

2012 Bioinspir. Biomim. 7046009

(http://iopscience.iop.org/1748-3190/7/4/046009)

View the table of contents for this issue, or go to the journal homepage for more

Download details:

IP Address: 31.222.247.71

The article was downloaded on 13/09/2012 at 22:04

Please note that terms and conditions apply. 


\title{
Towards a high-resolution flow camera using artificial hair sensor arrays for flow pattern observations
}

\author{
A M K Dagamseh ${ }^{1}$, R J Wiegerink, T S J Lammerink and G J M Krijnen \\ Transducers Science and Technology Group, $\mathrm{MESA}^{+}$Research Institute, University of Twente, \\ PO Box 217, 7500 AE, Enschede, The Netherlands \\ E-mail: a.m.k.dagamseh@ewi.utwente.nl
}

Received 4 June 2012

Accepted for publication 13 August 2012

Published 6 September 2012

Online at stacks.iop.org/BB/7/046009

\begin{abstract}
Flow-sensor arrays uncover the potential to measure spatio-temporal flow patterns rather than flow measurements at just a single point. We present in this paper the developments in design, fabrication and interfacing of biomimetic flow-sensor arrays, inspired by flow-sensitive organs (cerci) of crickets. For the purpose of high-resolution flow field visualization by our artificial hair flow-sensor arrays, various array-interfacing schemes are discussed and compared. Frequency division multiplexing (FDM) is shown to be an attractive method for efficient interrogation of capacitive array sensors. Using silicon-on-insulator technology with deep trench isolation structures, hair-based flow-sensors with differential capacitive read-out, arranged in single-chip arrays, have been successfully fabricated. FDM is implemented and used to interrogate individual hair sensors providing simultaneous real-time flow measurements from multiple hairs. This powerful approach is demonstrated by reconstruction of the field of a harmonic dipole field at the position of the hairs and by localizing this dipole source relative to the array elements.
\end{abstract}

(Some figures may appear in colour only in the online journal)

\section{Introduction}

\subsection{Array sensors}

In the past decades and in various disciplines, sensor arrays have become important research topics for researchers developing smart systems. Sensor arrays contain a plurality of discrete sensor elements each of which provides information about single- or multi-modal stimuli. The array composition and structure can be extensive in quantity and dimension. By fusing the data from all sensors, the array-information can transcend to be more than just a set of measurements, revealing source-information by spatiotemporal flow variations. Likewise, inter- and extra-polation of data from various sensors allow for estimates of stimuli at points not coinciding with the discrete sensors.

${ }^{1}$ Current address: Electronics Engineering Department, Hijjawi Faculty for Engineering Technology, Yarmouk University, Irbid, Jordan.
In the literature several examples of sensory array systems, in particular mechano-sensory arrays, have been reported. Applications in which such sensor arrays are used include finger-print sensors (Nigel 1999), image sensors (Aw and Wooley 1996), memory chips (Simoni et al 1995), etc. Many of these arrays have been equipped with e.g. capacitive sensors (Nigel 1999) and photo sensors (Bruijn et al 2004).

In biomedical applications, several medical diagnostic techniques take advantage of sensor arrays. The brain neurons' activities are recorded (electroencephalography) using arrays of electrodes in which the number, locations and strengths of the neural sources are estimated (Yildiz et al 2007). Artificial electronic tongues and noses have generally employed arrays of sensors. In such systems, arrays of micro-cantilevers are used to detect the presence of specific substances based on (electro-) chemical reactions which can be used later for automatic classification and recognition (Gardner and Bartlett 1992, Hauptmann et al 2000). 


\subsection{Biomimetic sensors}

In nature, animals can detect various events in their environment using a variety of stimuli, leading to perception of specific conditions relevant for their survival, e.g. finding food, reproduction and avoidance of predators (Shimozawa et al 1998). In these animals, ample examples of arrays of (highly sensitive) sensors can be found for which the fusion of the data retrieved by these sensors defines the function of the organ. Many mammalian senses are found in multisensory arrays; light receptors in the eye, smell and taste receptors in nose and tongue are some examples of such array systems. These sensor arrays are sensitive, versatile and intrinsically redundant. The large amount of data from all array elements is processed and the retrieved information is propagated through various levels in the nervous system to instigate further action as needed.

Biomimetics is a burgeoning field that examines principles and solutions for challenging environmental interaction problems as derived from biological examples. The added values in using bio-inspired sensor designs are mainly to surpass the performance and robustness of traditionally engineered sensory systems. This attains e.g. improvements in sensing accuracy, operational capability, reliability, size, robustness, costs and ease of use of such sensory system. Additionally, the availability of such biomimetic sensory systems helps scientists to understand nature (Steinmann et al 2006, Casas et al 2010). Imitating the principles from eyes to the camera (Ko et al 2008), whiskers of rodents to sensors for collision avoidance (Kima and Möller 2007), sonar system in bats to acoustic detectors (Reijniers and Peremans 2007) and cricket or fish hairs to artificial hair flow-sensors (Krijnen et al 2007, Dagamseh et al 2010) are examples of biomimetic sensors and sensory systems. The last example of cricket hairs forms the core of the work presented here.

\subsection{Artificial hair flow-sensors}

Biological hair sensor arrays are one of the most sensitive systems and appear widely in nature. The sensing hairs of crickets and the cilia-based lateral-line system of fish are examples of array-based sensory systems used to detect flows in air and water, respectively. A large mechano-sensory hairarray resides on the cerci of crickets, at the rear of their abdomen, forming the sensing part of a cricket's escape mechanism, e.g. to counteract spider-attacks (Shimozawa et al 1998). Typically, air movement due to the approaching predators causes the cricket to rapidly turn away from the stimulus. The large numbers of hairs, their mechanical properties and directivity and the array density result in an accurate and robust sensing system. This system enables the cricket to detect, localize and distinguish between various predators using the detected hydrodynamic airflow signatures (Shimozawa et al 1998).

Recently, the mechano-sensory hairs of crickets have become a common research topic for both biologists and engineers (Casas et al 2010, Krijnen et al 2007). Inspired by crickets and using advances in MEMS technology, several designs for single and arrays of artificial hair flow-sensors have

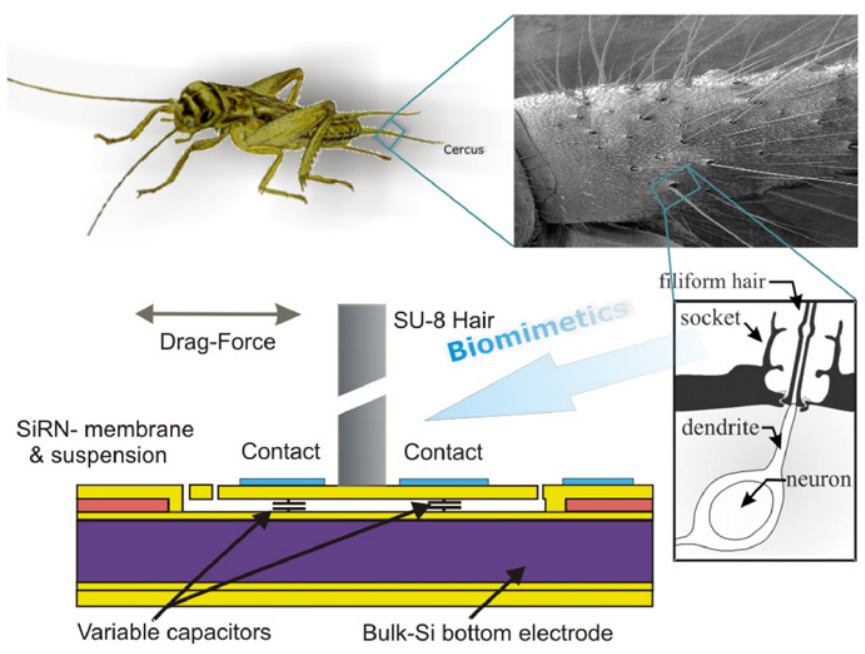

Figure 1. Schematic representation of a hair flow-sensor as inspired by crickets. (SEM close-up of a cricket's cercus courtesy of Professor J Casas, Institut de Recherche sur la Biologie de l'Insecte, Tours, France.)

been implemented successfully in various research groups (Dijkstra et al 2005, Fan et al 2002, Wang et al 2007, Bruinink et al 2009, Van Baar et al 2005). The research performed on these hair sensors has been mostly confined to the mechanical design, fabrication and characterization of (single) hair sensors (Fan et al 2002, Bruinink et al 2009). Figure 1 shows our artificial hair sensor along with its source of inspiration.

1.3.1. Sensing principle. Hair flow-sensors are fabricated using surface micro-machining technology to form suspended silicon nitride membranes with $\sim 1 \mathrm{~mm}$ long SU-8 hairs on top. The detection principle is based on differentially measuring capacitance changes between two electrodes deposited on top of the membrane and a common underlying electrode i.e. the silicon substrate. Due to viscous drag torque acting on the hair, the membranes tilt and in consequence the capacitors on both halves of each hair sensor change equally but oppositely. Two, mutually out-of-phase, alternating voltage sources (carrier signals at $\sim 1 \mathrm{MHz}$ ) are used to detect capacitance changes by the amplitude modulation (AM) signal. Subsequently synchronous demodulation is used to recover the original (baseband) airflow signals.

1.3.2. Fabrication. The hair flow-sensors' fabrication process starts with the deposition of a thin silicon nitride layer $(200 \mathrm{~nm})$ on a highly conductive silicon wafer, which forms the common electrode of the integrated capacitors. A $600 \mathrm{~nm}$ poly-silicon sacrificial layer is then deposited to define the capacitors' gap. The membrane and the torsion beams are formed by depositing and patterning a $1 \mu \mathrm{m}$ thick silicon rich silicon nitride layer. Subsequently, a $100 \mathrm{~nm}$ thick aluminium layer is sputtered onto the membrane and etched afterwards to form the top capacitor electrodes. The SU-8 hair is fabricated with two different diameters using a two-step photolithography process. Finally, the hair flow-sensors are released from the substrate by etching of the sacrificial polysilicon layer. Figure 2 shows a 3D schematic of the artificial 


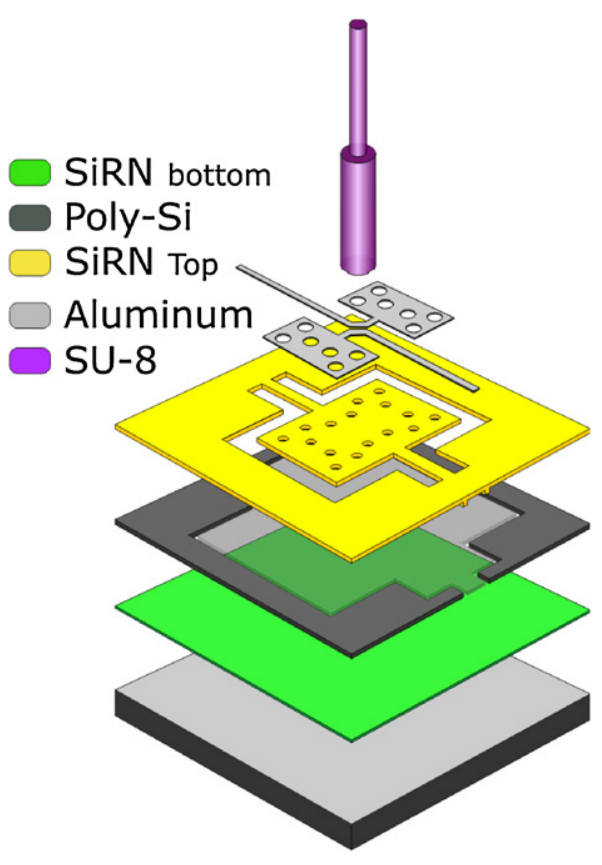

Figure 2. A 3D schematic representation of the artificial hair sensor structure as fabricated using MEMS technology.

hair flow-sensor geometry.

Previously, we have characterized the sensitivity of the hair flow-sensor to the airflow using laser vibrometry (Krijnen et al 2007) as well as capacitive measurements (Dijkstra et al 2005). Several essential design and fabrication parameters have been adjusted to optimize the detection limit of the hair sensors, e.g. using longer SU-8 hairs, smaller inter-electrode gaps and optimal membrane design (Dijkstra et al 2005, Bruinink et al 2009). In most of these designs, each hair flowsensor contains a group of 124 hairs connected in parallel with the same orientation to increase the total capacitance changes. An SEM image of a part of a hair flow-sensor, illustrating the array architecture, is shown in figure 3.

\subsection{Array addressing challenges}

Sensor arrays pose considerable technical challenges in the design, interfacing and subsequent signal processing. These challenges, specifically array interfacing, can limit the overall performance of the array system (especially) when the array task is to make real-time spatio-temporal pattern measurements. Such measurements can only be realized when each array element is interrogated individually and preferably continuously.

Generally, our capacitive hair flow-sensor arrays are constructed from rows and columns of conductive electrodes separated by dielectrics and arranged in a grid where the capacitances are located at the row-column intersections. The number of sensors depends on the area of interest and the resolution required. To achieve spatio-temporal flow measurements each hair sensor needs to be individually connected to its own acquisition channel. One method to form real-time pictures of airflow (using our hair sensors) is attained by acquiring multiple signals simultaneously by

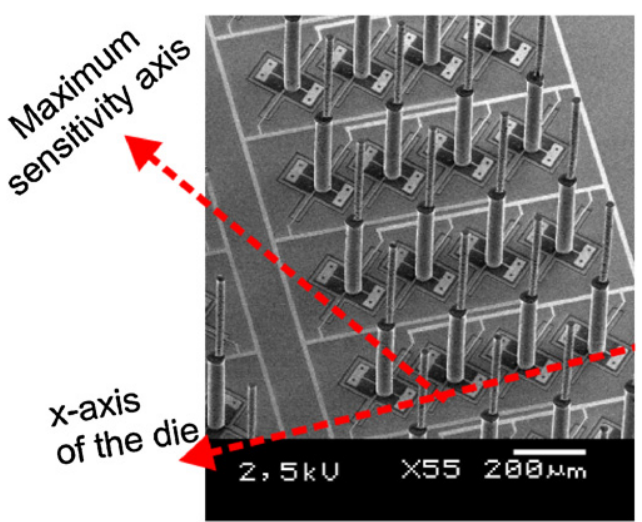

Figure 3. An SEM image for the artificial hair flow-sensor.

means of physically separated signal-carrying channels for each array element i.e. space division multiplexing (SDM). The advantage of this scheme is that all array elements are interrogated simultaneously and the performance (signalto-noise ratio (SNR), bandwidth, detection limit) of each interfaced hair sensor in the array shows no deterioration compared to its single hair-sensor equivalent. However, in large arrays technical challenges arise in making fast, parallel, accurate measurements while still maintaining a manageable number of read-out channels. The associated wiring complexity and required bond-pad real estate pose a large impetus for reduction of the number of connections. In our hair sensor, SDM would require a number of $3 \times n \times m$ connections, where $n$ and $m$ represent the number of columns and rows in the array, respectively, and the factor of 3 stems from the fact that each sensor needs two inputs and one output due to the differential capacitive measurement method. Hence, the main drawback of SDM is the overall system cost, limitations in available fabrication technology (for on-chip crossing of wires) and system complexity (number of signalcarrying channels which scales proportionally with the number of array elements). This makes it unattractive to use this scheme for large hair sensor arrays. Thus, sharing transmission channels between multiple sensors is a much-needed approach to reduce the number of interconnects.

As a pre-requirement for addressing large numbers of sensors, the prospective mechanisms should not lead to any deterioration in the performance of the individual hair sensors. The suitability of any addressing scheme is determined by its impact on the acquisition mechanism and trade-offs between sensor sensitivity, crosstalk, SNR and bandwidth. Additionally, the system cost, complexity of the architecture and the transmission speed of the signals must be considered. Here we will discuss the most promising addressing schemes for our hair flow-sensor arrays.

More specifically the main objectives of this work are

- realize a strong reduction of the number of connections calling for interconnects that are shared between the individual hair flow-sensor;

- solutions need to have all interfacing complexity offchip since our hair sensors have no on-chip electronic functionality; 
- the SNRs of the addressed hair sensors in the array should not appreciably differ from that of single hair sensors;

- maintaining minimum crosstalk between addressed sensors;

- the addressing complexity should be kept to a minimum; and

- the addressing scheme should preserve the sensors' bandwidth, which covers roughly dc to $1 \mathrm{kHz}$.

Bearing in mind our target for achieving live airflow measurements, this study answers several questions relevant to the bio-inspired hair flow-sensor arrays. What are the main interfacing mechanisms that can be implemented and how can they suit our hair sensor and array designs? What are the advantages and drawbacks of the various interfacing schemes? What are the main parameters, which significantly influence the performance of each interfacing scheme?

Additionally, we present, for the first time, single-chip flow-sensor arrays with simultaneous and separate read-out of individual hair sensors. The combination of these arrays with the proposed array-interfacing technique enables highresolution digital flow cameras, which form the basis for, e.g., flow pattern observation, categorization and recognition. Up to date the research performed on these hair sensors has been confined mostly to the hair mechanical design, fabrication and characterization. In this paper the key requirements for sensory array-interfacing and -addressing techniques with external circuits, to be applied on our hair flow-sensor arrays, are discussed and an implementation is demonstrated.

\section{Array addressing}

Event detection by sensing and observation of practical stimuli requires the measurement of multiple data, and sensor arrays are employed to meet these needs in a parallel and distributed fashion. However, performing assays on a large number of spatially distributed elements requires methods for multiplexing while rapid read-out of the sensors' signals is required to retain sufficient temporal information. Usually, interfacing large numbers of sensors is achieved by spatially wiring array elements or using multiplexing schemes, e.g. in the time or frequency domains, in order to reduce the number of connections. Frequency division multiplexing (FDM) (Chervenak et al 1999, Xu et al 2006) and, more often, time division multiplexing (TDM) techniques (Nelson and McMahon 1980, Wright and Porter 1992) are described in the literature to extract signals individually from an array of sensors.

TDM is used for example to multiplex an array of intensity-based optical sensors i.e. in an optical time-domain reflectometer (Nelson and McMahon 1980). In this system, the sensors are spatially distributed with different distances between the source and detector. A single pulse of appropriate duration at the input to the network produces series of distinct pulses at the output. These pulses represent time samples of the sensor outputs interleaved in a time sequence. The required duration of the input pulse is determined by the effective optical delay of the fibre connecting the sensor elements. Repetitive

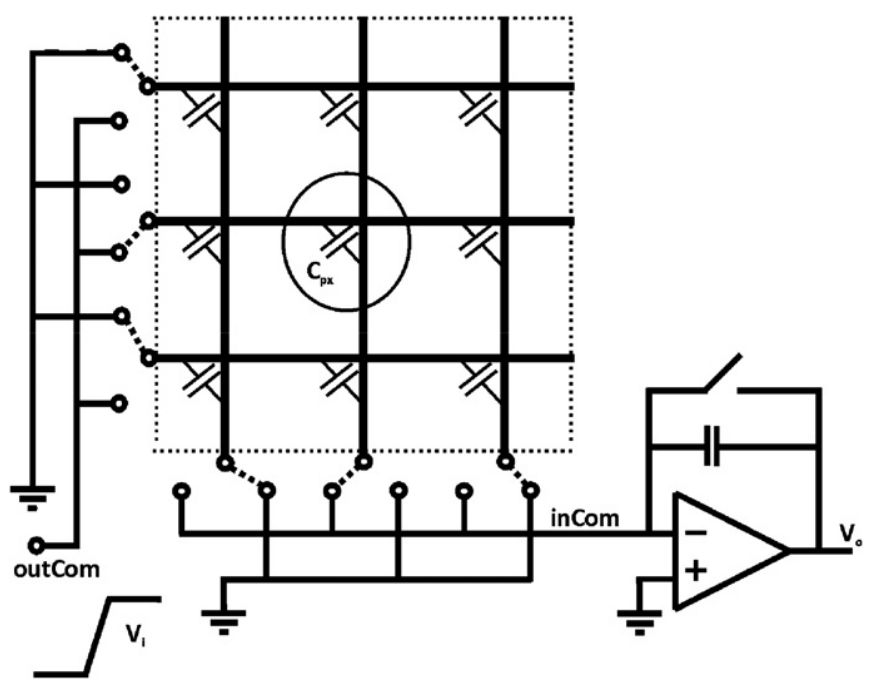

Figure 4. Principle of the TDM scheme as applied to a capacitive sensor array (adapted from Sergio et al (2002)).

pulsing of the system allows each sensor to be addressed by simple time-selective gating of the detector.

The TDM and FDM schemes have also been used for the acquisition of magnetic resonance images (MRI). TDM is used to acquire multiple images by multiplexing the signals from each coil to a single channel (Wright and Porter 1992). Using FDM, two signals from different RF coils encoded with different frequencies were combined to be transmitted to a high-speed analogue-to-digital converter (ADC) (Xu et al 2006). SQUID ${ }^{2}$ TDM is a well-developed method for reading out multiple transition edge sensors (TES) in a single amplifier channel. In this system the number of SQUIDs is equal to the number of detectors but only one SQUID is turned on at a time i.e. TDM (de Korte et al 2003, Doriese et al 2007).

The TDM scheme has also been demonstrated for reading capacitive sensor arrays. This system consists of circuits for selecting specific column and row lines to activate a single array element. The selected element is connected to an inverting amplifier while the deselected elements are connected to a reference voltage (Sergio et al 2002). Such a method can be applied to any capacitive sensor array such as load cells' sensory array systems (Zwijze 2000), capacitive finger-print sensors (Nigel 1999) and image sensors (Sartori et al 1995, Aw and Wooley 1996, Theuwissen 2003). Figure 4 illustrates the basic principle of TDM as applied to a capacitive sensor array.

Another example of interfacing array of sensors is image sensors. Image sensors typically contain charge coupled devices (CCD) or complementary metal-oxidesemiconductor devices in which each pixel element is at the crossing of a specific row and column. In this system, the measurement operation begins by resetting all of the pixels. Next, the absorbed light energy generates charges in each individual pixel within the array, which represents the electrical replica of the optical image. Further, the sequential read-out unloads charges from each pixel element. The readout circuit, which uses internal decoders and addressing

2 SQUID: Superconducting QUantum Interference Device. 


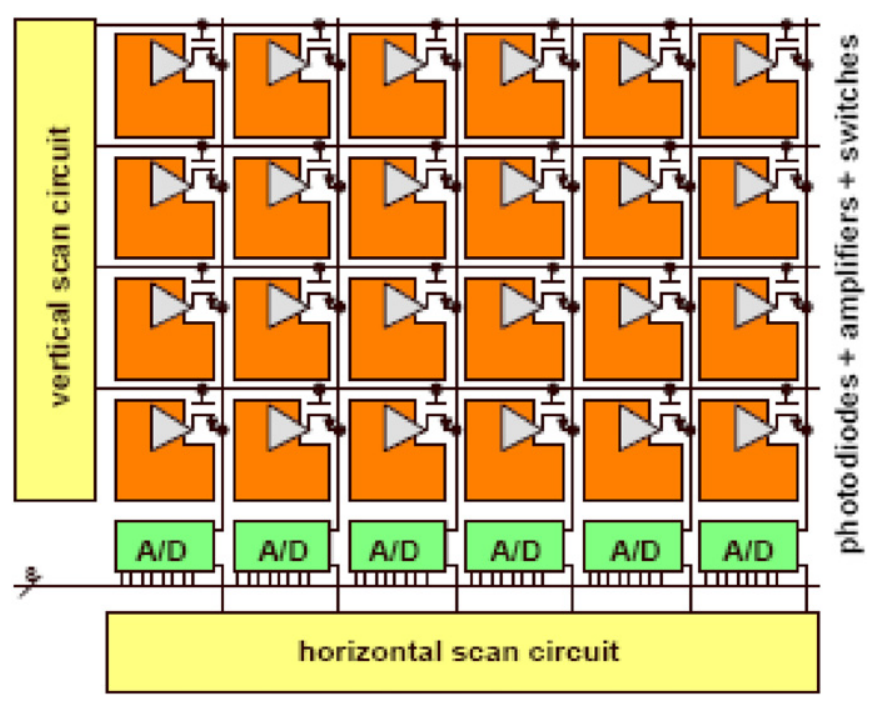

Figure 5. Schematic representation of an image sensor showing pixel arrays and electronic control circuit (Theuwissen 2003).

control signals, scans the whole pixel array sequentially. One pixel is read at a time while the other pixel charges are stored in capacitors associated with and parallel to the photodiode of each pixel, waiting for signal acquisition. After completing the read-out cycle the sensor is again reset. Various technological and design techniques have been considered to achieve fast, compact and high charge-transfer efficiency while reducing crosstalk and noise at different levels of chip integration. Figure 5 shows a CCD image sensor which contains an array of pixels and control circuits. Various integrating levels for the electronics have been implemented to reduce noise, costs, complexity and scanning time (Sartori et al 1995, Theuwissen 2003).

The basic idea of multicarrier modulation was introduced and patented in the mid 60s by Chang (Chang 1970). This technique has been widely used in communication systems and data transmission. Array addressing using FDM techniques has been demonstrated in fibre optic array systems and imaging arrays of micro-calorimeters using a TES and SQUID devices (Dandridge 1994, Yoon et al 2001, Kiviranta et al 2002). In this system, array elements are probed with alternating currents at distinct frequencies. When the TES absorbs signal power, its resistance changes cause modulation of the amplitude of the alternating probing current. The output signals are summed and connected to a single SQUID amplifier. At the electronics side the individual sensor signals are retrieved by a bank of frequency-selective demodulators.

The FDM scheme has also been used in radar-based frequency-modulated continuous wave (FMCW) techniques (Inaudi 2003). The processing produces a beat frequency associated with each sensor element, allowing frequency demultiplexing of the outputs, with the magnitude of the beat signal as a measure of the reflectance of the sensors. The FMCW technique enables the sensors to be located at different spatial locations to be discriminated.

A hybrid scheme is another possibility to multiplex sensor arrays. In this scheme, FDM and TDM are used to combine the beneficial attributes of both (McGarrity et al 1995, Liu and

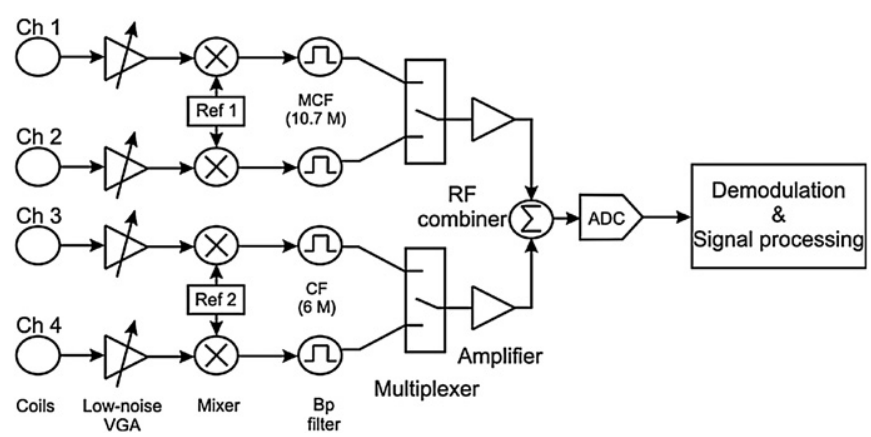

Figure 6. Block diagram representing four-channel parallel acquisition of a MRI system using the hybrid scheme (adapted from Reintsema et al (2008)).

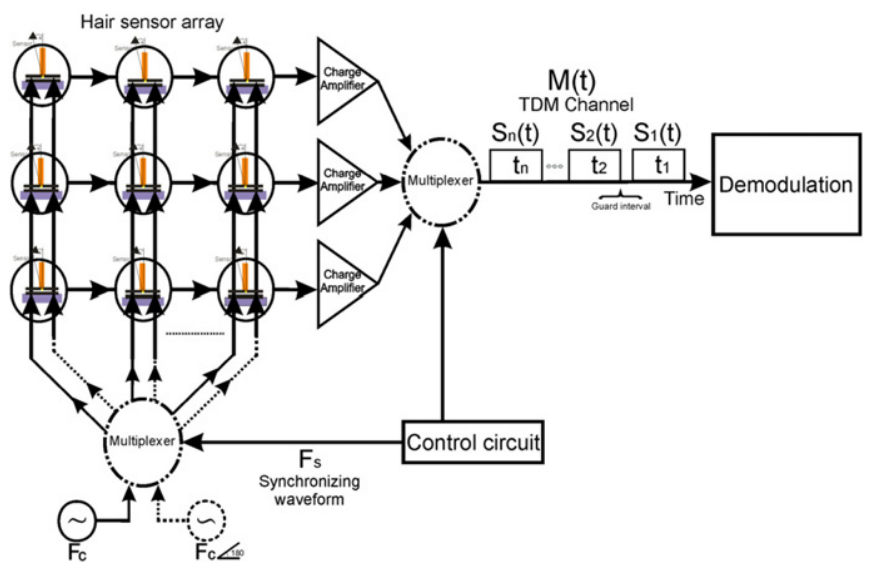

Figure 7. Block diagram of a possible TDM implementation for addressing hair sensor arrays.

Li 2008, Reintsema et al 2008). The hybrid scheme has been used to multiplex arrays of reflective interferometric sensors (McGarrity et al 1995), TES (Reintsema et al 2008) and MRI sensors (Liu and Li 2008). In a TES system, a firststage of SQUID amplifiers, whose signals are time-division multiplexed into the second-stage SQUID, employ FDM to more efficiently utilize the available bandwidth. In this method, combination of time and frequency multiplexing is used for parallel acquisition of MRI images (see figure 6). As a result of that only a single ADC and a commercial multicarrier receiver are needed, which results in a less complex system.

\subsection{TDM}

TDM is one of the possible schemes to reduce the number of input and output connections (Nelson and McMahon 1980, Xu et al 2006). A possible TDM scheme applied to our hair sensor arrays, shown in figure 7 , is achieved by scanning array elements with one multiplexer connected off-chip to the column side and one multiplexer on the row side. The two multiplexers determine the element to be measured. All acquired signals originating from multiple array elements share a single output-channel over time. 
With TDM, the number of signal-carrying channels ${ }^{3}$ and their interface connections can be greatly reduced when compared with SDM. However, there are some limitations especially for large sensor arrays.

- Perfect synchronism of the multiplexer control waveforms at the column and row sides is essential to prevent crosstalk. This requires the implementation of control circuits which add more complexity to the system.

- Loss of parallelism ${ }^{4}$ inherent to the sequential scan of TDM due to the increase of sensor dead-time ${ }^{5}$ as the number of array elements increases. This hampers the essential requirement in making live measurements.

- The array bandwidth (i.e. the number of multiplexed sensors times the bandwidth of each sensor) increases linearly with the number of sensors. On the other hand, to maintain a given array bandwidth the bandwidth available to each hair sensor in an array of $N$ sensors can only be $1 / N$ of the array bandwidth at maximum. Also, maintaining a given sensor bandwidth imposes limitations on the TDM scheme for an increasing number of sensors. The acquisition time for each element reduces inversely proportional to the number of array elements. For instance, a sensor with a bandwidth $\triangle f \mathrm{~Hz}$ requires $1 / 2 \triangle f$ seconds' maximum acquisition time intervals (i.e. a $2 \triangle f \mathrm{~Hz}$ sampling rate). So an array of $N$ sensors can only allow for $1 / 2 N . \triangle f$ seconds' acquisition time per sample thereby degrading the individual SNR. In other words, increasing the array size $N$ times requires reduction of the acquisition time of each single element by a factor of $N$ to preserve the same sensor bandwidth according to

$$
T_{\text {element }}=\frac{T_{\text {array }}}{N},
$$

where $T_{\text {element }}$ is the acquisition time per array element and $T_{\text {array }}$ is the acquisition time for the entire array system. Consequently, the individual SNR deteriorates by $N^{1 / 2}$.

- The switching circuits are an extra source of noise for the array system, specifically if they are integrated at the early stages of the acquisition system. The TDM scheme employs switches for scanning the array, generating considerable noise. Thus, the scope for integrating both the scanning and acquisition systems is reduced.

- In our hair sensor, the generated AM signals are in the $\mathrm{MHz}$ range, which requires both high frequency multiplexing and high sampling rate. Hence, the multiplexer has to scan over all array elements in cycling times that are smaller than one over twice the bandwidth of each hair element (Nyquist criterion). Using the TDM scheme imposes severe bandwidth requirements for the interfacing circuits and system bandwidth.

- The crosstalk between sensors is exacerbated due to the electrical coupling through small capacitances between the switch channels.

3 Signal-carrying channels represent the outputs of the array. In the case of SDM each sensor has its own signal-carrying channel while in TDM the entire array has a single carrying channel.

4 Parallelism denotes the ability to acquire signals from various sensors at the same time instance (i.e. making live measurements).

5 Dead-time of a sensor is the time when the sensor is not activated (not connected to the interfacing electronics channel).

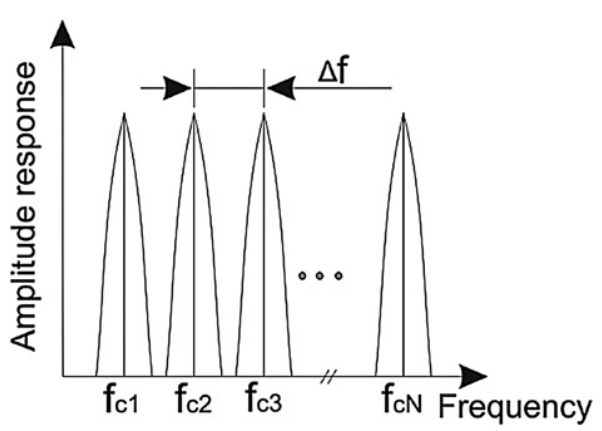

Figure 8. Schematic representing the spectrum of the multicarrier signal as attained using FDM.

- There is a time lag between the detected signals from successively interrogated sensors, since each signal is measured at a slightly different time. This will result in timing errors up to the sampling interval in the spatiotemporal measurements.

\subsection{FDM}

Based on the above discussions, obviously, using TDM is not ideal for acquiring instantaneous array measurements. A different array-multiplexing scheme has to be used to overcome the disadvantages and limitations of TDM and SDM, especially when dealing with large numbers of sensors. We present alternative schemes based on FDM in which array signals are multiplexed in the frequency domain.

FDM is a well-known scheme in communication and data transmission. It refers to a type of modulation in which several carrier signals with different frequencies are modulated by several band-limited data signals to form one wide-band, multicarrier signal. Thus, the available bandwidth is divided into a number of 'frequency-channels'. If the multicarrier signal has to be converted into a digital form, the maximum number of FDM channels that can be combined is limited by the Nyquist bandwidth of the ADCs i.e. the ratio of the Nyquist bandwidth of the ADC to the maximum frequency in the multicarrier signal. Therefore, higher speed ADCs are required to achieve more FDM channels. Figure 8 shows the principle of FDM.

A major advantage of FDM is sharing of the available bandwidth for signal transmission off-chip. This makes FDM inherently a parallel process without affecting the acquisition time of individual sensors i.e. the SNR is maintained irrespective of array size. Additionally, in the time domain no guard intervals are required for signal separation; therefore, neither switches nor multiplexing waveforms (as in TDM) are needed. As a result there is no switching noise and less hardware and fewer connections to perform the same task are needed. In effect, FDM can be executed as a pure analogue scheme without any switching logic involved in the signal channel.

Figure 9 shows a schematic representation of a possible FDM scheme as applied to our hair sensor array. Using FDM, a bank of oscillators is used to generate bi-phasic ( $180^{\circ}$ out-ofphase) sinusoidal signals i.e. carriers. These carriers are used to probe the array columns, which form the bottom electrodes of the capacitive hair sensors (see the fabrication process of hair 


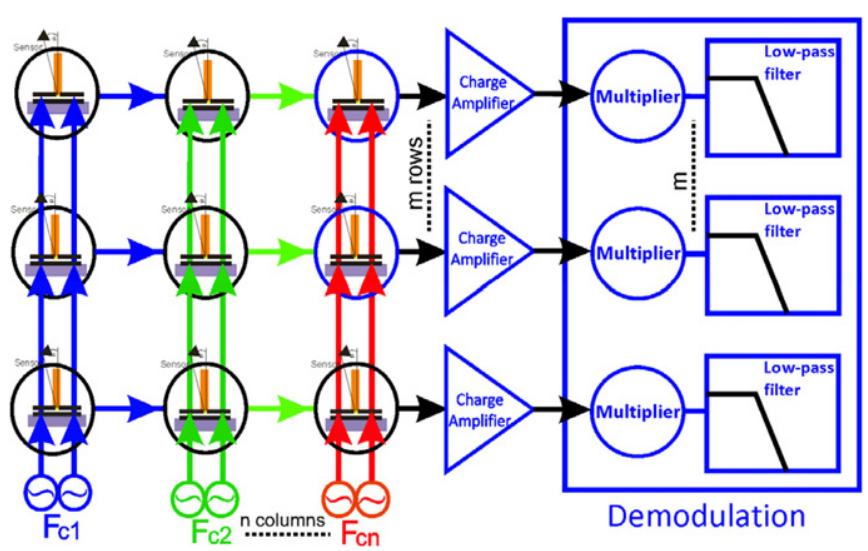

Figure 9. Multiplexing a hair flow-sensor array using the FDM technique.

arrays dedicated for FDM shown in section 3.1). Capacitance changes due to the imposing airflow modulate the amplitude of the carrier signals according to

$$
S(t)=A \cos \left(\omega_{c} t\right) H(t),
$$

where $S(t)$ represents the transmitted AM signal from each hair sensor, $A$ the amplitude of the carrier signal, $\omega_{c}$ the carrier frequency and $H(t)$ the flow signal from each hair sensor.

At the row side and due to the use of different carrier frequencies, the resulting signals appear as mutually frequency shifted signals allowing them to be combined (equation (3)) and transmitted through a single wire while staying distinguishable. The summation point not only consists of the common electrode of all hair sensors in one row but also a connection to the input of a charge amplifier.

$$
q_{k}(t)=\sum_{j=1}^{n} S_{k, j}(t),
$$

where $k$ represents the row number, $q_{k}(t)$ the row signal stream of row $k, S_{k, j}(t)$ the signal of the sensor located at column $j$ and row $k$ and $n$ is the total number of columns in the array.

Multiplying the composite output with a specific carrier frequency, used at the columns' side, the information from each individual hair sensor can be retrieved again in the baseband (i.e. demodulation process). Alternatively, the output signals can be converted to a digital form for more advanced processing operations.

Considering our requirement of achieving real-time measurements, the FDM scheme is favourable as a method of interfacing artificial hair sensor arrays. In our hair sensor array the FDM addressing scheme has advantages for the following.

- Making spatio-temporal measurements, which requires a parallel measurement process. Such a system opens opportunities to measure, characterize and eventually recognize specific spatio-temporal flow signatures by the simultaneous extraction of individual sensor signals.

- Reducing hardware complexity and integration costs (i.e. reducing the number of interconnects by a factor of $3 \cdot n \cdot m /(2 \cdot n+m)$ and the charge amplifier count by a factor of $n$, where $n$ and $m$ represent the number of array columns and rows, respectively). In FDM, the reduction in system complexity scales linearly with the number of array elements, and the advantage of FDM is more realized for large array elements. The optimal size, for the least number of connections, is when the number of rows is twice the number of columns (for the case of hair sensor arrays).

- Maintaining the SNR, since the acquisition time for individual elements is constant, independent of array size.

- Array scalability i.e. the array size can be extended without deteriorating the SNR.

- Minimizing possible crosstalk, since neither synchronizing waveforms nor switches are required.

\subsection{Optimal addressing scheme}

The hybrid scheme, which involves implementing FDM and TDM together to take advantage of both schemes, can be used in array addressing. For example, columns are activated using FDM while signals at the row side are multiplexed using TDM. Combining FDM and TDM improves, to some extent, the multiplexing gain of the sensor array i.e. use of the available bandwidth and hardware efficiently. However, optimization for the number of hardware channels and bandwidth is required to determine the optimal contribution of each scheme for each case separately. With the assistance of our previous knowledge of array design, sensor design and carrier frequency range it is clear that TDM is not the optimal solution since it uses only a fraction of time to connect a sensor to the read-out channel thereby limiting the sensor bandwidth, parallelism and reducing the SNR.

In line with our conditions and requirements and based on the above discussions (taking into account the impossibility of electronics integration on-chip), the FDM mechanism represents the most favourable addressing scheme to be implemented with our artificial hair flow-sensor arrays. FDM allows addressing and reconstruction of the original signal from individual sensors separately, which makes it feasible to measure spatio-temporal flow patterns across arrays of hair flow-sensors.

Summing up, the FDM approach maintains the sensor and array bandwidth as it is with SDM while the bandwidth is scaling with the number of array elements in TDM. The number of array connections required to implement SDM is huge and can be tremendously reduced with FDM and TDM. A comparison between the previously presented main array-interfacing schemes based on our requirements is shown in table 1. The numbers are based on single hair-sensor requirements with a bandwidth of $B_{s}$ and array size of $(n \times m)$ hair sensors.

The following sections show the implementation of the FDM scheme in interfacing artificial hair sensor arrays. The design and fabrication process of the hair sensor are modified, from the previous sensor design, allowing for the fabrication of single-chip arrays. The results are shown for arrays made of $5 \times 4$ hair elements but the extension to larger arrays is straightforward. 

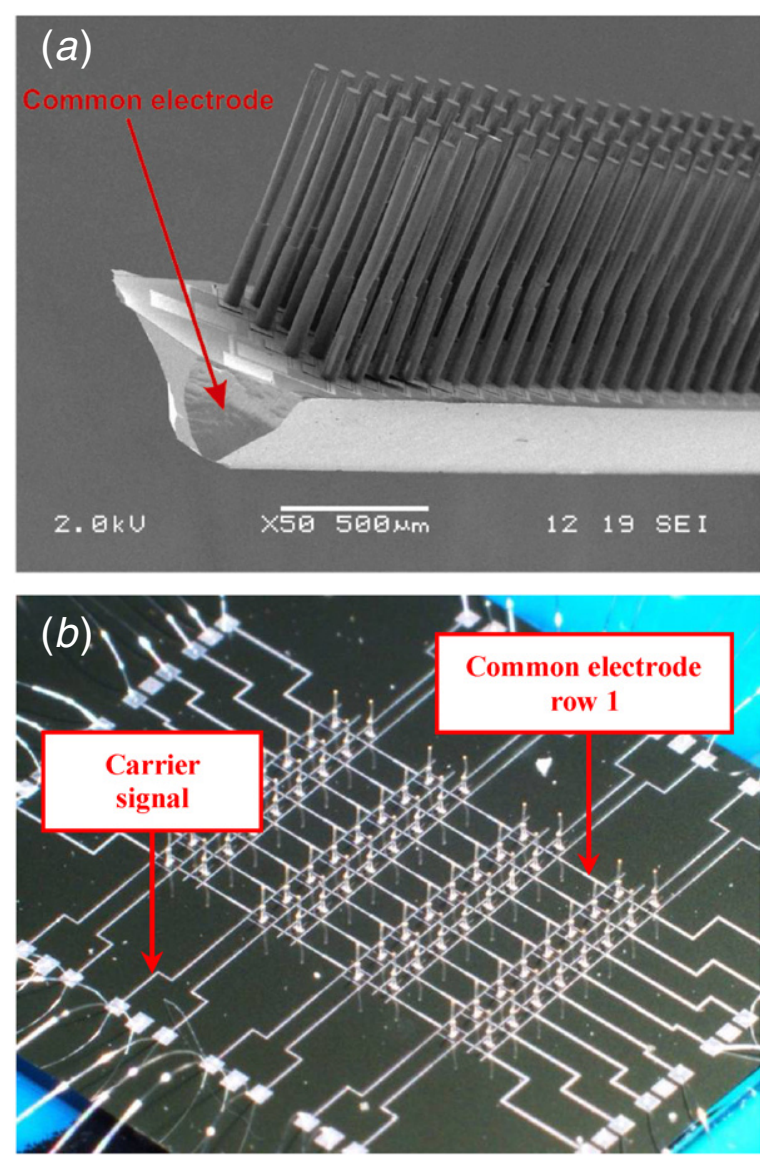

Figure 10. (a) An SEM image of a previous artificial hair flow-sensor array showing the grouping principle (many hairs arranged in parallel with the substrate as common electrode). (b) Image of a single-chip hair sensor array dedicated to FDM and consisting of single-hair elements with pairs of sensors arranged perpendicular to each other.

Table 1. Comparison between main array-interfacing schemes as applied to our hair sensor arrays.

\begin{tabular}{|c|c|c|c|}
\hline & SDM & TDM & FDM \\
\hline Crosstalk & + & - & \pm \\
\hline Scalability & - & - & + \\
\hline Interfacing & Continuous & Intervals & Continuous \\
\hline $\begin{array}{l}\text { Simultaneous } \\
\text { measurements }\end{array}$ & Real-time & Delayed & Real-time \\
\hline $\begin{array}{l}\text { Electronics } \\
\text { complexity }\end{array}$ & Very complex & Complex & Less complex \\
\hline $\begin{array}{l}\text { Bandwidth per } \\
\text { channel }\end{array}$ & $B_{s}$ & $(n \times m) \times B_{s}$ & $B_{s}$ \\
\hline $\begin{array}{l}\text { Number of } \\
\text { connections }\end{array}$ & $3(n \times m)$ & $2 n+m$ & $2 n+m$ \\
\hline $\begin{array}{l}\text { SNR factor } \\
\left(\frac{S N R_{\text {single }}}{S N R_{\text {addressed }}}\right)\end{array}$ & 1 & $(n \times m)^{1 / 2}$ & $1^{\mathrm{a}}$ \\
\hline
\end{tabular}

${ }^{a}$ Taking into account perfect carrier signals and low common electrode resistance.

\section{Experiments}

\subsection{Hair sensor array fabrication}

Previously we have shown the design and fabrication of highdensity flow-sensitive hair sensor arrays (Krijnen et al 2006)

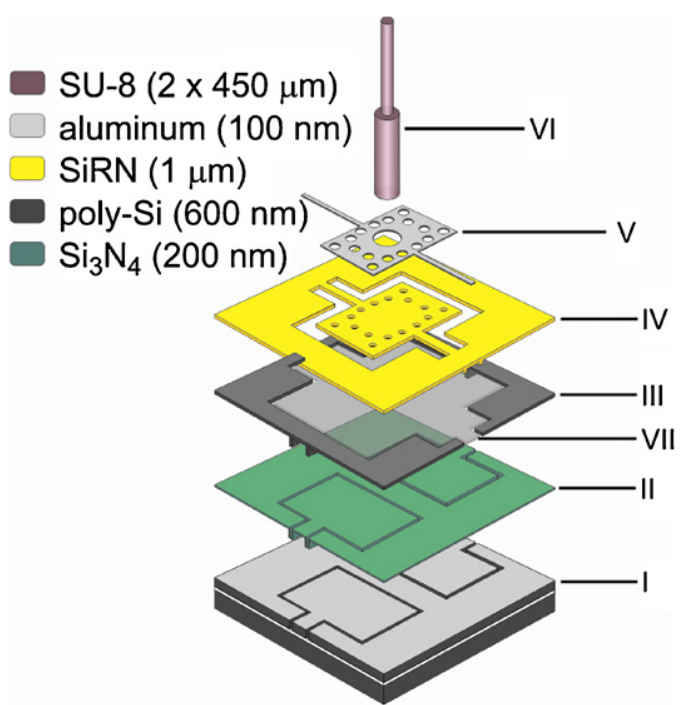

Figure 11. Schematic representation of the new artificial hair sensor design.

(see figure 10(a)). Signals from a group of hair sensors were combined to increase the SNR (i.e. increasing the common capacitance changes but averaging out noise from similar but independent hairs). However, in that (previous) sensor design the underlying silicon substrate forms the common electrode for the capacitors and integrates the signals of all hair sensors. This prevents addressing hair sensors independently and thereby hampers single-chip-based arrays. Therefore, the design of the hair sensor arrays has been adapted to implement FDM.

Here we look into the possibilities to make high-density single-chip hair sensor arrays which can be interfaced using a capacitive measurement method comparable to what we have done before and which can be made in a compatible fabrication process. The fabrication process of the single-chip hair sensor arrays (see figure 10(b)) is shown in figure 11. Arrays of artificial hair flow-sensors were fabricated in rows and columns with single hair sensors as basic array elements. The use of silicon-on-insulator (SOI) wafer technology allows us to isolate the sensing electrode of each hair element requiring primarily a redesign of the electrode system. This assists in interrogation of each individual hair sensor by a differential capacitive measurement technique requiring only two antiphase carrier signals. In the new hair sensor design the carrier signal electrodes are defined in the silicon device layer (using deep trenches for insulation) while the common electrode for the output signal is implemented by an aluminium layer on the top silicon nitride layer (including the sensor membranes). The common electrode of all single-hair elements for each row are connected together (i.e. facilitating FDM). Additionally, due to a reduction of parasitics and noise, this design improves the single-sensor detection limit to reach about $1 \mathrm{~mm} \mathrm{~s}^{-1}$ using a bandwidth of $3 \mathrm{kHz}$ as shown in (Dagamseh 2011).

Figure 11 illustrates the fabrication process of the hair sensor arrays. The fabrication process starts by etching deep insulation trenches into the $25 \mu \mathrm{m}$ thick device layer of the SOI wafer down to the $\mathrm{SiO}_{2}$ layer by directional reactive-ion 


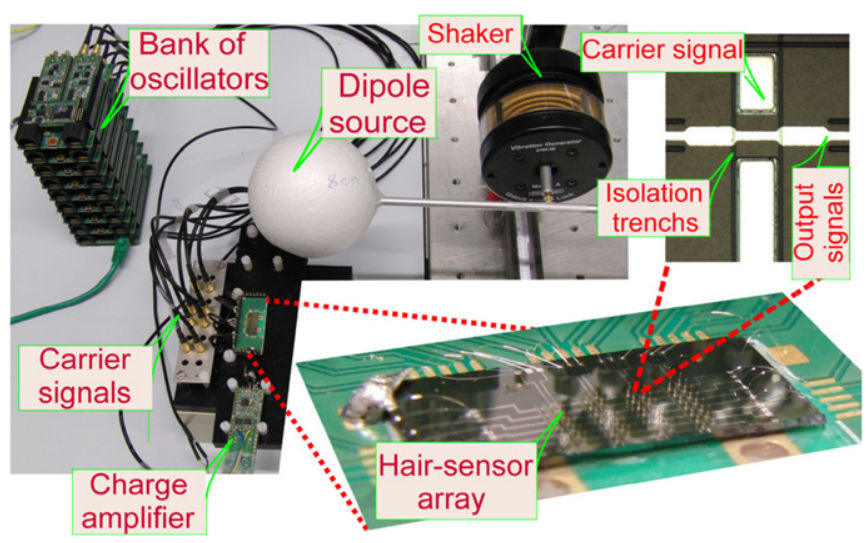

Figure 12. Photograph of the measurement setup and sensor arrays. Isolation trenches of the carrier electrodes are also shown.

etching (RIE), thus forming the bottom electrodes (figure 11-I). Subsequently a thin nitride $\left(\mathrm{Si}_{3} \mathrm{~N}_{4}, 200 \mathrm{~nm}\right)$ layer and a thick poly-Si layer $(1400 \mathrm{~nm})$ are deposited by low-pressure chemical vapour deposition (LPCVD) to protect the bottom electrodes during later sacrificial layer etching (SLE) and to completely fill the isolation trenches separating the bottom electrodes, respectively (figure 11-II/III). Two wet oxidation runs $\left(1150{ }^{\circ} \mathrm{C}, 120 \mathrm{~min}\right)$ and successive etching in BHF are applied to reduce the poly-silicon layer to $600 \mathrm{~nm}$, the eventual gap distance. Then insulation trenches are patterned into the poly-Si by RIE (figure 11-III). Next a $1 \mu \mathrm{m}$ thick SiRN layer is deposited by LPCVD and etched by RIE to form the actual membranes and torsion beams (figure 11-IV). A $100 \mathrm{~nm}$ thick aluminium layer is sputtered and patterned to form the sensor top electrode (figure 11-V). This layer also reduces the series resistance of the carrier signal electrode when it is deposited on top. This is followed by two sequential spinning-exposure procedures of two $450 \mu \mathrm{m}$ thick SU-8 layers to fabricate $900 \mu \mathrm{m}$ long hairs (figure 11-VI). Finally the structure is released by etching the sacrificial poly-silicon layer (figure 11-VII) using $\mathrm{XeF}_{2}$ gas.

\subsection{Measurement setup}

Figure 12 shows the experimental setup and the sensor array used to demonstrate the FDM scheme. An array of $(5 \times 4)$ artificial hair sensors was addressed using FDM to simultaneously measure signals from individual hair sensors. The 20 sensors were addressed using 4 times 2 carrier frequencies at the inputs of the columns and 5 row outputs to the charge amplifiers, giving a total of 13 interconnects rather than the 60 that would have been required for SDM. A bank of oscillators (made of direct digital synthesis signal generators on dedicated printed circuit boards) was used to generate the carrier signals as applied to the array columns. Along the rows, the AM signals were combined and connected off-chip to one charge amplifier per row. The array functioning was tested using a harmonic dipole field, since it is well described in the literature (Lamb 1910). A sphere with a radius of $5 \mathrm{~cm}$ was harmonically driven at $30 \mathrm{~Hz}$ (as stimulus) to examine the performance of the hair sensor array in measuring flow fields and perform source localization.

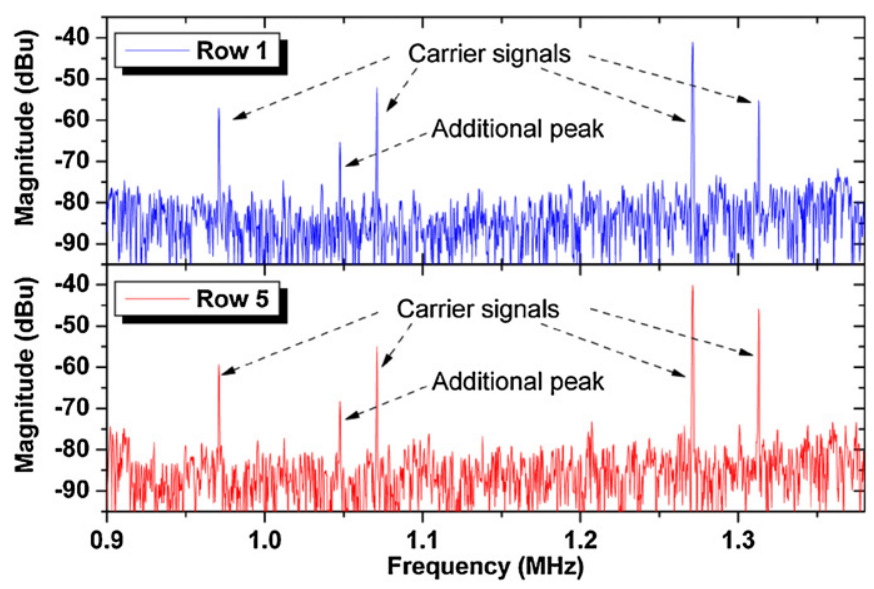

Figure 13. FFT spectrum of AM signals from two rows at the output of the charge amplifiers while employing FDM to an array of $5 \times 4$ hair sensors.

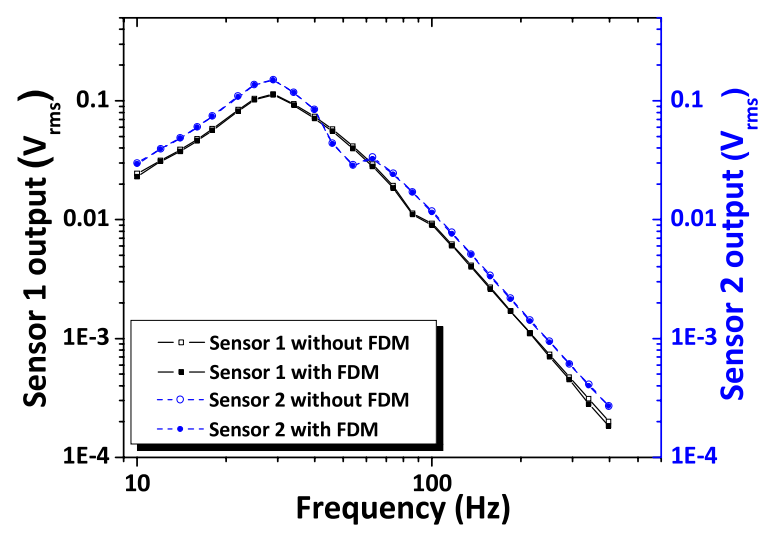

Figure 14. Frequency response of two hair sensors (located in the same row but in two different columns within a single-chip array) measured without and with FDM.

\subsection{Results}

We were able to simultaneously measure airflows for each hair sensor while using FDM. Due to the availability of the hardware channels, TDM (with relay system) was used to switch between the array rows such that four hair sensors in each row were simultaneously measured using FDM. Figure 13 shows the frequency spectrum of the composite signal measured at the output of the charge amplifier representing the four AM carrier signals for two (arbitrarily) selected FDM channels (rows 1 and 5).

First the performance of the FDM array-interfacing scheme as applied to our hair sensor arrays was investigated using loudspeakers as airflow source. The synchronous demodulation process was performed using a $300 \mathrm{~Hz}$ lowpass filter. Outputs of the hair sensors, with and without FDM, were measured and compared. Figure 14 shows the frequency response of two hair sensors (two hair sensors in the same row were chosen $)^{6}$ The results show virtually identical frequency response for each of the hair sensor irrespective of the use of FDM.

${ }^{6}$ Note that these graphs show 'raw data' which includes the loudspeaker characteristics (so the curves do not present the sensor transfer function). 


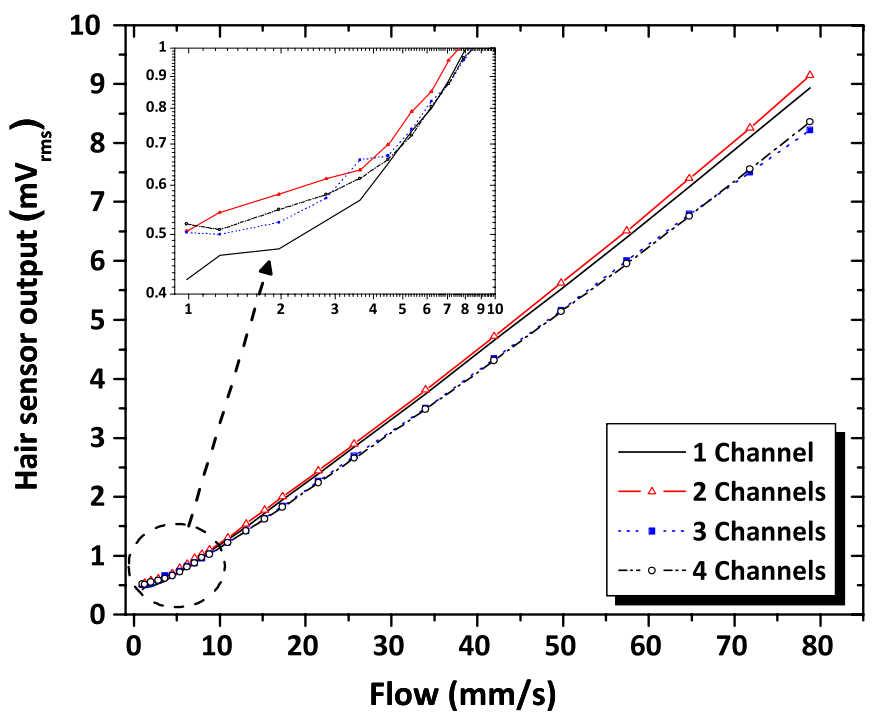

Figure 15. Effect of the application of FDM on the voltage output of a single hair sensor when an increasing number of channels is activated.



Figure 16. Flow field measurement versus dipole position simultaneously detected by four hairs in one row. The separation between peaks matches with the hair sensor separation.

Figure 15 illustrates the effects of FDM on the voltage output of a hair sensor (measured at $80 \mathrm{~Hz}$ ) when activating successively 1, 2 and 3 hair sensor located in the same row. The results indicate that the performance of the addressed hair sensor does not deteriorate significantly by the application of FDM.

As demonstration for successful measurement of airflow fields using hair sensor arrays, the dipole fields were measured by different hairs and for a systematically varied position of the vibrating sphere (virtual lateral line). Afterwards the positions of the dipole source (relative to the array elements) were determined. Figure 16 represents the dipole fields detected by different hair sensors located in one row by means of this virtual lateral line (Dagamseh et al 2010). The shift in peak positions represents the column separation distance between sensors and precisely matches the physical design distance between the elements $(2 \mathrm{~mm})$.

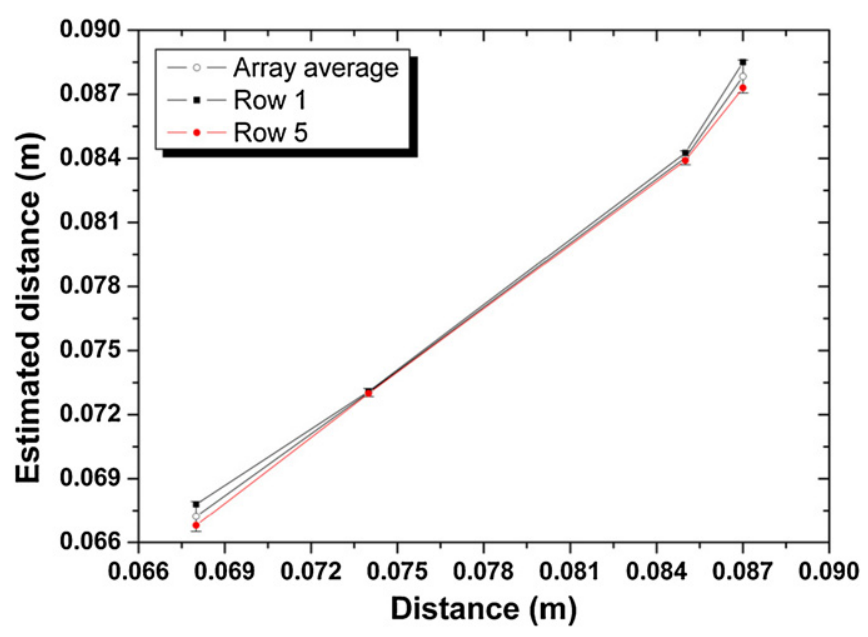

Figure 17. Dipole source localization using a hair sensor array while applying FDM.

The source localization process, using either the signals as averaged over row 1 or averaged over row 5, as well as the signals averaged over both rows are shown in figure 17. The distance between the source and the array is determined according to the methods described in Dagamseh et al (2010). A clear linear relation between the real and estimated distances is observed.

\section{Discussion}

In the new hair sensor design, some aspects in connection to the application of FDM for array interfacing have to be considered. Firstly, the combination of the series resistance and cable parasitics (from the carrier-signal source to the sensor capacitor) can pose limitations on the bandwidth and therefore the upper limit of the carrier frequencies. Hence, the maximum frequency and minimum frequency spacing between adjacent channels (necessary to reduce crosstalk) limit the number of columns that can be multiplexed. To overcome this, the series resistance of carrier signal electrodes is reduced by depositing an additional aluminium layer on top. The resistance of the carrier electrodes is in the range of $1 \mathrm{k} \Omega$ thereby nearly matching the resistance of the common electrode (300-400 $\Omega$ ). Secondly, if the impedance of a connection to a summation point (the input of a charge amplifier) is not equal to zero the summed current signal will produce a voltage drop. Consequently, each hair sensor is not only probed by its own carrier frequency but also by the carriers of neighbouring hairs. This generates crosstalk in which each hair sensor modulates the amplitude of multiple carriers, i.e. cross-modulation. The crosstalk amplitude depends on the impedance value at the specific carrier frequencies. To limit this effect, the impedance of the common electrode is kept small using aluminium for signal-carrying electrodes. Thirdly, nonlinearities of the carrier-signal generators cause additional frequency components that may interfere with neighbouring carrier channels (see additional peak in figure 13). If one of the carrier signals of neighbouring channels is carelessly selected to coincide at one of these additional 
peaks, crosstalk between FDM components occurs. As a result, the hair sensor's output would not only contain a modulated signal at its intended carrier frequency, but also frequency components at neighbouring channels, possibly modulated by neighbouring sensors (i.e. two carriers for a single hair sensor). Experimentally, the amplitudes of some of the demodulated signals were found to decrease when the carrier signal of one of the neighbouring channels was disconnected. To overcome this, the carrier frequencies were chosen appropriately to be free from additional frequency components from neighbouring channels.

The presented results show that array-wide we can measure the artificial hair sensor rotations as caused by spheremovement induced airflows. This confirms the successful application of the FDM addressing technique to our hair sensor array. As a consequence we are able to reduce the number of interconnects considerably. In the demonstrated array the reduction was from 60 interconnects to 13 (4 times 2 carrier frequencies input along the columns and 5 rows output to the charge amplifiers). The measurements confirm that the application of FDM does not significantly affect the performance of an addressed hair sensor, neither its frequency response nor its linearity. The results presented in figure 14 show very small deviations in frequency response of a hair sensor with and without FDM. On the other hand, when probing additional sensors in the same row reduction in the voltage response of a hair sensor is observed while it has almost no effect on noise level (see figure 15). This can be attributed to the limitation of multiplier input amplitudes: as the number of addressed hair sensors is increased, the voltage level at the input of the multiplier increases and shows saturation and other nonlinear effects. This poses limitations on the number of hairs that can be multiplexed in one channel. Proper selection of high-performance multipliers or performing the demodulation process in the digital domain can improve the FDM method in this respect.

The reconstructed virtual lateral-line responses prove that each hair element faithfully reflects the dipole field at its position while employing FDM. It demonstrates the hair sensor array's ability to probe individual hair sensors independently and afterwards to accurately localize the positions of dipole sources, relative to either each hair element or to the entire array. Consequently, it is demonstrated that FDM makes it possible to carry out simultaneous measurements with singlechip arrays consisting of multiple independent hair sensors. These achievements, in combination with the use of highly sensitive single hair sensors arranged in single-chip arrays, enable us to accurately measure the projection of the flow field at the position of the array. Various processing tasks can be implemented taking advantage of these simultaneous measurements of signals from the entire array.

Importantly, FDM is not limited to addressing of capacitive sensor arrays, and also (piezo-, thermo-, magneto-) resistive or inductively probed arrays can take advantage of it. In a resistive scheme, the variable resistors are probed with alternating voltages with distinct frequencies at the column side and the signals are summed at the row side. This makes FDM a universal array-interfacing scheme that allows reducing system complexity considerably.

\section{Conclusions}

In conclusion, this paper detailed an important step of our work towards 'flow cameras' based on biomimetic hair flowsensor arrays. By gathering signals from individual sensor elements, it is possible to obtain spatio-temporal images of airflows over hair sensor arrays. Several schemes for interfacing individual array elements in array-settings have been discussed. It is found that the use of FDM is favourable for interfacing our capacitively interrogated hair sensor arrays. Unlike TDM, FDM is free from propagation delays and synchronization errors. Therefore, channel acquisition techniques and hardware for FDM are not as complex as for TDM. Further, FDM is rather scalable to larger array structures without reduction of the acquisition time and deterioration of the SNR of the individual sensors. SOI-based fabrication technology in combination with FDM has opened up the possibility for simultaneous interfacing of multiple sensors in single-chip arrays. This adds new dimensions to flowimaging with potential applications in e.g. flow monitoring and robotics.

In future work, airflow patterns will be investigated with larger numbers of hair sensors. Such systems will allow us to determine specific information, such as source position and direction of movement, by measurement of spatio-temporal flow-profiles and recognition of specific flow signatures of moving objects. One possible technique for signature detection is the use of artificial neural networks. Consequently, this further expands the biomimetic approach (which has been already used in the design of the artificial hair flow-sensor) to the data processing stage of the array signals towards more perceptive use of flow fields.

\section{Acknowledgments}

This research was made possible by grants from the Customized Intelligent Life-Inspired Arrays (CILIA) project funded by the Future and Emergent Technologies arm of the IST programme of the EU and from a Bio-EARS Vici grant from the Dutch Technology Foundation (STW/NWO).

\section{References}

Aw C H and Wooley B A 1996 A $128 \times$ 128-pixel standard-CMOS image sensor with electronic shutter IEEE J. Solid-State Circuits 31 1922-30

Bruijn M P et al 2004 Development of an array of transition edge sensors for applications in X-ray astronomy Nucl. Instrum. Methods Phys. Res. A 520 443-5

Bruinink C M, Jaganatharaja R K, de Boer M J, Berenschot E, Kolster M L, Lammerink T S J, Wiegerink R J and Krijnen G J M 2009 Advancement in technology and design of biomimetic flow-sensor arrays Proc. IEEE MEMS (Italy) pp 152-5

Casas J, Steinmann T and Krijnen G 2010 Why do insects have such a high density of flow-sensing hairs? Insights from the hydromechanics of biomimetic MEMS sensors J. R. Soc. Interface 7 1487-95

Chang R W 1970 Orthogonal frequency division multiplex data transmission system US Patent Document Number 3488445 
Chervenak J A, Irwin K D, Grossman E N, Martinis J M, Reintsema C D and Huber M E 1999 Superconducting multiplexer for arrays of transition edge sensors Appl. Phys. Lett. 74 4043-5

Dagamseh A M K 2011 Bio-inspired hair flow sensor arrays: from nature to MEMS PhD Thesis University of Twente, Enschede, The Netherlands

Dagamseh A M K, Lammerink T S J, Kolster M L, Bruinink C M, Wiegerink R J and Krijnen G J M 2010 Dipole-source localization using biomimetic flow-sensor arrays positioned as lateral-line system Sensors Actuators A $162355-60$

Dandridge A 1994 The development of fiber-optic sensor systems Proc. SPIE 2360 154-61

De Korte P A, Beyer J, Deiker S, Hilton G C, Irwin K D, MacIntosh Nam S W, Reintsema C D, Vale L R and Huber M E 2003 Time-division superconducting quantum interference device multiplexer for transition-edge sensors Rev. Sci. Instrum. 74 3807-15

Dijkstra M, Van Baar J J, Wiegerink R J, Lammerink T S J, De Boer J H and Krijnen G J M 2005 Artificial sensory hairs based on the flow sensitive receptors hairs of crickets J. Microelectron. Microeng. 15 S132-8

Doriese W B et al 2007 14-pixel, multiplexed array of gamma-ray microcalorimeter with $47 \mathrm{eV}$ energy resolution at $103 \mathrm{keV}$ Appl. Phys. Lett. 90193508

Fan Z, Chen J, Zou J, Bullen D, Liu C and Delcomyn F 2002 Design and fabrication of artificial lateral line flow sensors J. Microelectron. Microeng. 12 655-61

Gardner J and Bartlett P N 1992 Sensors and Sensory Systems for an Electronic Nose (Dordrecht: Kluwer)

Hauptmann P, Borngraeber R, Schroeder J and Auge J 2000 Artificial electronic tongue in comparison to the electronic nose- - state of the art and trends Proc. 54th Annual IEEE Int. Frequency Control Symp. (USA) pp 22-9

Inaudi D 2003 State of the art in fiber optic sensing technology and EU structural health monitoring projects 1st Int. Conf. on Structural Health Monitoring and Intelligent Infrastructure (Tokyo, Japan)

Kima D and Möller R 2007 Biomimetic whiskers for shape recognition Robot. Auton. Syst. 55 229-43

Kiviranta M, Seppä H, van der Kuur J and de Korte P 2002 SQUID-based read-out schemes for microcalorimeter arrays AIP Conf. Proc. 605 295-300

Ko H C et al 2008 A hemispherical electronic eye camera based on compressible silicon optoelectronics Nature 454 748-53

Krijnen G J M, Dijkstra M, Van Baar J J, Shankar S S, Kuipers W J, De Boer R J H, Altpeter D, Lammerink T S J and Wiegerink R 2006 MEMS based hair flow-sensors as model systems for acoustic perception studies Nanotechnology 17 S84-9

Krijnen G, Lammerink T, Wiegerink R and Casas J 2007 Cricket inspired flow-sensor arrays Proc. 6th IEEE Conf. on Sensors (IEEE Sensors) (Atlanta, GA, USA) pp 539-46
Lamb H 1910 The Dynamical Theory of Sound (London: Edward Arnold)

Liu Y and Li G 2008 FTDM receiver for parallel acquisition of MRI Electron. Lett. 44 454-6

McGarrity C, Chu B and Jackson D 1995 Multiplexing of Michelson interferometer sensors in a matrix array topology Appl. Opt. 34 1262-8

Nelson A R and McMahon D H 1980 Passive multiplexing system for fiber optic sensors Appl. Opt. 19 2917-20

Nigel D Y 1999 Capacitive sensing array device US Patent Document number 5952588 A

Reijniers J and Peremans H 2007 Biomimetic sonar system performing spectrum-based localization IEEE Trans. Robot. 23 1151-9

Reintsema C D et al 2008 A TDMA hybrid SQUID multiplexer J. Low Temp. Phys. 151 927-33

Sartori A, Maloberti F, Simoni A and Torelli G 1995 A 2-D photosensor array with integrated charge amplifier Sensors Actuators A 46 247-50

Sergio M, Manaresi N, Tartagni M and Canegallo R 2002 Method of reading a capacitive sensor and related integrated circuit $U S$ Patent Document number 7755683

Shimozawa T, Kumagai T and Baba Y 1998 Structural scaling and functional design of the cercal wind-receptor hairs of cricket J. Comp. Physiol. A 183 171-86

Simoni A, Torelli G, Maloberti F, Sartori A, Plevridis S and Birbas A N 1995 Single-chip optical sensor with analog memory for motion detection IEEE J. Solid-State Circuits 30 800-5

Steinmann T, Casas J, Krijnen G and Dangles O 2006 Airflow sensitive hairs: boundary layers in oscillatory flows around arthropod appendages J. Exp. Biol. 209 4398-408

Theuwissen A 2003 Lecture notes TUDelft

Van Baar J J, Dijkstra M, Wiegerink R, Lammerink T S J, De Boer R and Krijnen G J M 2005 Arrays of cricket-inspired sensory hairs with capacitive motion detection Proc. 18th IEEE Int. Conf. on MEMS (Miami, USA) pp 646-9

Wang Y H, Lee C Y and Chiang C M 2007 A MEMS-based air flow sensor with a free-standing micro-cantilever structure Sensors 7 2389-401

Wright S W and Porter J R 1992 Parallel acquisition of MR images using time multiplexed coils Electron. Lett. 28 71-2

Xu Q, Wang H, Xu Z and Li G 2006 Frequency domain multiplexing for parallel acquisition of MR images Electron. Lett. 42 326-7

Yildiz G, Duru A D and Ademoglu A 2007 A comparative study of localization approaches to EEG source imaging Proc. IEEE /NIH Life Science System and Applications Workshop (LISA, Bethesda, MD) pp 156-9

Yoon J, Clark J, Gildemeister J, Lee A, Myers M, Richards P and Skidmore J 2001 Single superconducting quantum interference device multiplexer for arrays of low-temperature sensors Appl. Phys. Lett. 78 371-3

Zwijze A F 2000 Micro-machined high capacity silicon load cells PhD Thesis University of Twente, Enschede, The Netherlands 